\title{
Perioperative Pain Management in the Critically III Patient
}

\author{
Michal Czernicki ${ }^{1}$ - Sreekumar Kunnumpurath ${ }^{2}$ - William Park ${ }^{3}$. Anamika Kunnumpurath ${ }^{4}$ - Gopal Kodumudi ${ }^{5}$. \\ Jing $\mathrm{Tao}^{3} \cdot$ Vijay Kodumudi $^{6} \cdot$ Nalini Vadivelu $^{3} \cdot$ Richard D. Urman $^{7}$
}

Published online: 11 April 2019

(C) Springer Science+Business Media, LLC, part of Springer Nature 2019

\begin{abstract}
Purpose of Review The assessment and management of perioperative pain in an intensive care setting is complex and challenging, requiring several patient-specific considerations. Administering analgesia is difficult due to interacting effects of pre-existing conditions, interventions, and deviation from standard levels of expressiveness of pain. A significant part of this complexity also arises from the reduced capacity of critically ill patients to fully communicate the severity and nature of their pain. We provide an overview of pharmacological approaches and regional techniques, which can be employed alongside the management of anxiety and sleep, to alleviate pain in the critically ill patients in the perioperative period. These interventions require additional assessments unique to critical care, yet achieving pain relief for improving clinical outcomes and patient satisfaction remains a constant.

Recent Findings The latest research has found that the development of standardized mechanisms and protocols to optimize the diagnosis, assessment, and management of pain in the critically ill can provide the best outcomes. The numerical rating scale, critical care pain observation criteria, and behavior pain scale has shown higher reliability to accurately assess pain in the critically ill. Most importantly, preemptive analgesia and the emphasis on early pain control —in the perioperative setting, ICU, and post-discharge - are crucial in minimizing chronic post-discharge pain. Finally, the multimodal approach is still found to be the most effective. This includes pharmacological treatments, regional nerve block, and epidural techniques, as well as alternative methods that are cheap, safe, and easily available. All these together have shown to help control pain, provide psychological support, and prevent long-term co-morbidities in the critically ill.

Summary Largely, pain in the critically ill patient is still a very complex issue that requires appropriate diagnosis, assessment, and management of the pain itself and treating all the underlying co-morbidities as well. Many different factors makes it challenging, especially the difficulty in communicating with an ICU patient. However, by looking at the patient as a whole, treating pain early with the multimodal approach, there seems to be some promising results in improving outcomes. It has shown that the improved outcomes in critically ill patients in the perioperative period seen with optimized pain management and ICU can shorten hospital stays, decreased inpatient costs, and limit the use of limited resources.
\end{abstract}

This article is part of the Topical Collection on Other Pain

Michal Czernicki

mczernicki@yahoo.com

Sreekumar Kunnumpurath

skunnumpurath@gmail.com

Anamika Kunnumpurath

anamika.kunnumpurath.13@ucl.ac.uk

Gopal Kodumudi

gopal.kodumudi3577@cnsu.edu

Vijay Kodumudi

vkodumudi@uchc.edu

Richard D. Urman

rurman@bwh.harvard.edu
1 Consultant Anaesthetist, Nottingham University Hospital, Derby Road, Nottingham NG7 2UH, UK

2 Consultant in Pain Management, Epsom and St. Helier University Hospitals, Wryth Lane, Carshalton SM5 1AA, UK

3 Department of Anesthesiology, Yale University, 333 Cedar Street TMP3, New Haven, CT 06520, USA

4 Medical School, University College London, Gower Street Bloomsbury, London WC1E 6BT, UK

5 California Northstate School of Medicine, 9700 West Taron Drive, Elk Grove, CA 95757, USA

6 University of Connecticut School of Medicine, 263 Farmington Avenue, Farmington, CT 06030-1905, USA

7 Department of Anesthesiology, Perioperative and Pain Medicine, Brigham and Women's Hospital, Boston, MA 02115, USA 
Keywords Pain $\cdot$ Management $\cdot$ Critically ill $\cdot$ Intensive care unit $\cdot$ Regional anesthesia $\cdot$ Multimodal

\section{Introduction}

Pain is one of the most common symptoms experienced by critically ill patients in the post-surgical intensive care units. It is estimated that about $80 \%$ of patients have moderate to severe pain and $71 \%$ of intensive care unit (ICU) survivors recall experiencing pain post-discharge from the ICU [1]. Despite the significance of controlling pain in the critically ill, pain is often overlooked [2•], and given less priority than other lifethreatening aspects of the clinical conditions. For example, Puntillo et al. points out that less than $25 \%$ of patients receive preemptive analgesia before painful procedures [3]. This demonstrates that not enough emphasis is placed on the importance of preemptive analgesia and preventing central sensitization. Therefore, in the last decade, Barr et al. demonstrates the importance of appropriate diagnosis and management of pain as a key factor in improving post-surgical ICU patient outcomes and post-discharge pain [2•]. This body of literature has promoted the development of standardization mechanisms and protocols to optimize the diagnosis, assessment and management of pain in the critically ill [4].

Uncontrolled pain in the critically ill patient is expressed and experienced in a multitude of ways and can lead to complications. It can be directly associated with the primary pathology, painful procedures, or the psychological distress that comes with being critically ill and undergoing surgery. Uncontrolled painful stimuli have been demonstrated to lead to acute stress responses including endocrine secretion and psychological stress [5, 6]. It also increases the incidence of nosocomial infections, the need for mechanical ventilation, and the ICU length of stay [7]. The psychological implications of uncontrolled pain are also significant within the intensive care unit in perioperative period. Individuals who retain memories of frightening pain experiences are at increased risk of developing post-traumatic stress disorder, which affects 34 $38 \%$ of discharged patients from the ICU [8]. Using protocols to titrate sedation in mechanically ventilated patients and monitoring sedation level can help decrease the emotional stresses of recall of painful procedures and mechanical ventilation [9].

Furthermore, Woolf et al. explains that the management of this uncontrolled pain actually begins in the perioperative setting. For example, the notion of preemptive analgesia and preventing central sensitization is becoming an important method to decrease chronic post-surgical pain [10]. By controlling and managing pain early on in the perioperative setting and into the ICU, better clinical outcomes and overall patient satisfaction have been reported. A recent analysis of published data from four separate studies demonstrated, for example, that pre-emptive analgesia to control pain in patients undergoing lung transplantation was associated with decreased duration of mechanical ventilation, length of stay in the intensive care unit, and improvements in other respiratory parameters such as progression with using the incentive spirometer [11]. In a recent prospective study involving patients in the ICU post-cardiac surgery, a specialized pain management protocol was developed to adequately train staff members on appropriate pain management and to assess pain. For example, the study explains the implementation of a new pain assessment tool in which nurses can use a standardized method to assess pain and delivery the appropriate analgesic therapy accordingly. Although this particular study did not demonstrate a difference in length of stay in the ICU on duration of mechanical ventilation, a reduction in the frequency of intractable pain was demonstrated [12].

The cumulative evidence suggesting the urgent importance of early and adequate pain diagnosis, assessment, and management has led the American College of Critical Care Medicine (ACCM) to recently publish revised clinical practice guidelines for the sustained use of sedatives and analgesics in the critically ill adults [2•]. This review highlights the most recent advances in the management of pain in patients with surgical and medical critical illness, focusing on pharmacological approaches, regional anesthetic techniques, and alternative therapies.

\section{Pathophysiology of Pain in ICU}

The physiological pathways that result in the sensation of pain are well researched and understood. The patient senses painful stimuli via the afferent pain pathway. This includes the release of inflammatory mediators resulting from tissue injury, leading to subsequent nociceptor stimulation and pain impulse transmission via the spinothalamic tract to the reticular activating system (RAS), thalamus, and somatosensory cortex, where the localization and meaning of pain occur. Under normal circumstances, the sensation of pain would cause the patient to take steps to prevent further noxious stimuli $[13 \cdot, 14]$.

The pain pathway is often altered in critically ill patients in that the verbal or purposeful responses may be inhibited in this vulnerable group of patients. Critical illness, muscle relaxants, and sedation are among the many reasons ICU patients are unable to adequately communicate further noxious stimuli. As a result, pain assessments also become difficult, which lead to frequently inadequate analgesia and the increased risk of development of chronic pain [10]. 
In addition, critical illness, surgical insult, or tissue injury initiate release of local inflammatory mediators including bradykinin, substance P, prostaglandins, potassium, histamine, and serotonin. These mediators may subsequently produce a heightened sensitivity to stimuli in the area of injury (hyperalgesia) or a perception of pain to normally nonnoxious stimuli (allodynia). This can ultimately lead to central sensitization and chronic post-surgical pain. Thus, the critically ill patients can experience pain similar to chronic pain patients $[10,13 \cdot, 15]$. Therefore, this notion preemptive analgesia and vigilantly controlling acute pain early on in the critically ill and perioperative setting become essential in the prevention of chronic pain into the ICU and post-discharge.

\section{Causes of Pain in ICU}

Critically ill patients may experience pain from the underlying illness or injury, from surgery, or from other non-surgical interventions. Pain may also result from a variety of noxious stimuli present in the ICU setting including invasive monitoring (i.e., arterial lines, central venous catheters), therapeutic devices (i.e., positive pressure ventilation, hemofiltration, nasogastric feeding tube), routine physical therapy (i.e., passive limb movement, endotracheal suction), or routine nursing care (i.e., turning).

Among critically ill post-surgical patients, it is often the type of surgery that may contribute to increased pain in the ICU. For example, thoracotomies, large abdominal surgeries, and nephrectomies, among other surgeries involving large incisions, are likely to increase pain. In addition to the nature of surgery, the degree of critical illness may make systemic therapies, such as opioids or epidurals less appropriate due to hemodynamic instability, compounding postoperative pain.

Certain medications used in surgically critically ill patients may also contribute to pain. For example, corticosteroids and non-depolarizing muscle relaxants may cause polyneuropathies or myopathies, which may in turn contribute to pain $[16,17 \bullet]$. For example, patients that require emergent intubation in the ICU with succinylcholine (RSI induction) can lead to severe myalgias and worsen their initial state of pain. Subsequent invasive procedures, transport, patient turning, new medications, and all the things involved in the ICU stay can further exacerbate and worsen their pain.

Managing pain in the critically ill is complicated by many psychological factors. Fear and anxiety are commonly reported occurrences in this group $[13 \cdot 18]$ with up to $43 \%$ of all patients in ICU reporting anxiety during their admission [19॰]. The commonness of anxiety may be attributed to several different factors, such as uncertainty surrounding the underlying illness, clinical interventions, and noxious environmental triggers [20 ${ }^{\bullet}$. Pain that is not sufficiently controlled can also contribute to a generalized sympathetic response, which may precipitate sleep and appetite disruption, elevating anxiety states [20•]

\section{Assessment of Pain}

Assessment of pain in the surgical ICU patient may be challenging due to sedation, delirium, mechanical ventilation, inotropic therapy, and muscle relaxation. It is estimated that less than $40 \%$ of nursing staff routinely assess pain in non-verbal ICU patients [14]. Routine assessment of pain in intensive care is crucial as it is an easy and low-cost intervention which improves outcomes including reduction in need of ventilator support and the length of ICU stay [21, 22].

According to ACCM, self-reporting pain scales are the gold standard in pain assessment in critically ill patients. Numerical rating scale (NRS) in vertical format is the most discriminative and feasible self-reporting scale in critically ill patients [23]. Unfortunately, the majority of patients are unable to self-report pain, and therefore, a behavioral pain scale is required to assess pain appropriately [24]. The role of vital signs in the measurement of pain is very limited as they poorly correlate with severity of pain [25].

Multiple behavioral pain scales are commonly used. However, only a few of them are validated and those include Critical Care Pain Observational Tool (CPOT) and Behavioral Pain Scale (BPS).

CPOT is based on four domains: facial expression, movements, muscle tension, and compliance with ventilator. Meta analysis of CPOT demonstrated higher reliability and validity compared to the other pain scales in non-verbal critically ill patients [24]. BPS focuses on three domains: facial expression, movement of upper limbs, and compliance with ventilator. BPS has been shown to be valid and reliable pain scale. However, it has high inter-rater variability [24].

\section{Treatment}

The management of pain is particularly challenging in critically ill surgical patients due to multiple factors. Most of these patients are semi-conscious, non-communicative, or both [26]. Pain is often underestimated and inadequately treated as a result. Conversely, pain levels based upon physiological parameters may be overestimated; however, patients should be presumed to have pain and many different treatment modalities should be offered and provided whenever the patient is unable to help in their pain assessment [27].

\section{Pharmacological Treatment}

The ideal analgesic should have a rapid onset, rapid offset, lack of accumulation, with no side effects or toxicity. Currently, no single agent with all these characteristics exists [16]. Therefore, pain levels, response to medication, and side effects should be monitored frequently. It is largely agreed that 
a pain rating of 3 or less out of 10 , or 2 or less out of 5 , is a desirable analgesic goal $[27,28]$. These are, however, patientspecific and would depend upon the clinical situation.

Regardless, pain in critically ill patients should be recognized and treated early. According to the World Health Organization (WHO) Analgesic Ladder, the three-step method to treating pain is to first utilize non-opioids, then mild opioids, and if refractory to all, strong opioids. However, opioids are often the first-line treatment for non-neuropathic pain in the ICU setting. All common intravenous opioids, including fentanyl, hydromorphone, and morphine, are considered equally effective. However, pharmacokinetics and pharmacodynamics properties based on healthy volunteers may not apply to critically ill patients. Meperidine should be avoided, as it may cause neurotoxicity (62).

The most prominent adverse effect of many opioid-based medications, such as morphine and fentanyl, is respiratory depression, but numerous other effects must also be considered, such as opioid-induced immunosuppression. Pathological mechanisms of immunosuppression include direct opioid effect on the immune system or an indirect effect on catecholamine and glucocorticosteroid secretion. Acute exposure to morphine inhibits lymphocytes T, B, NK, and macrophage migration $[29,30]$. Immunosuppression due to chronic exposure to opioids is possibly less relevant due tolerance to opioid-induced immunosuppression. In contrast, tramadol has immunostimulant properties and possibly should be the drug of choice for immunocompromised patients [31].

Opioid-naïve patients who have received moderate to highdose opioid analgesia for more than one week are prone to acute opioid withdrawal [16]. This can manifest in symptoms of drug craving, nausea, abdominal pains, and muscle aches, whereas signs may include fever, hypertension, tachycardia, vomiting, diarrhea, lacrimation, sweating, tremor, delirium, and seizures. These signs may be nonspecific in the critically ill non-verbal patient, and clinicians should have a low index of suspicion for withdrawal phenomena.

On the other hand, treatment of choice for patients with neuropathic pain in ICU is usually a combination of gabapentin or carbamazepine with opioids. Gabapentin and pregabalin are analogues of gamma-aminobutyric acid (GABA), and they bind to alpha-2 delta subunits of voltagegated calcium channels. Gabapentin significantly reduces pain scores and opioid consumption in neuropathic pain related to Guillain-Barre syndrome [32].

Non-steroidal anti-inflammatory drugs (NSAIDs) inhibit cyclooxygenase and subsequently production of prostaglandins. Multiple meta-analyses showed that NSAIDs reduce postsurgical pain from severe and moderate to mild in $50-60 \%$ of patients. However, their use in ICU is limited due to risk of bleeding, renal failure, and congestive cardiac failure [22].

Lidocaine, used as an intravenous infusion, is an approved treatment for neuropathic pain. Lidocaine infusion started prior to surgery and continued for 4 to $24 \mathrm{~h}$ postoperatively has been found to be effective in reducing postoperative pain and opioid consumption, and to reduce in-hospital stay [33, 34]. Lidocaine infusion could potentially be used in critically ill patients prior to any painful stimulus. However, studies are lacking and this requires further evaluation. Additionally, lidocaine plasma level above $5 \mathrm{mcg} / \mathrm{mL}$ is toxic. Therefore, if used, this should be monitored in ICU patients. Also, liver dysfunction can inhibit lidocaine metabolism.

Ketamine is a $N$-methyl-D-aspartate (NMDA) receptor antagonist. Its use has been studied in the peri-operative period. In patients who underwent spinal surgery, ketamine infusion significantly reduced pain scores and opioid consumption compared to a controlled group [35, 36]. Neuropsychiatric complications of ketamine were rarely reported and present in less than $5 \%$ of patients in sub-anesthetic doses.

Acetaminophen, or paracetamol, is a central inhibitor of cyclooxygenase and is an important adjunct to treat mild to moderate post-surgical pain. It is also an effective anti-pyretic agent that can be used in the short-term treatment of fever in critically ill patients. As it is metabolized in the liver, it should be used with caution in patients with liver dysfunction or those with low body weight. This medication can be delivered in multiple routes such as oral, rectal, or IV administration in the perioperative setting.

\section{Regional Techniques and Peripheral Nerve Blocks}

Regional anesthetic techniques are valuable methods in decreasing pain in postoperative critically ill patients. Peripheral nerve blocks not only improve peri-operative pain following extremity surgery and trauma but also reduce opioid consumption. Regional anesthesia as part of multimodal analgesia can improve overall outcome after major orthopedic surgery [37], including reducing the length of ICU stay. Beneficial effects can be maintained with continuous infusion peripheral nerve block [38].

Epidural infusion decreases the risk of myocardial infarction, respiratory complications, and gastrointestinal and renal dysfunction in patients following abdominal aortic aneurysm repairs [39]. Benefits of epidural infusion are considered to be higher for high-risk surgical patients [40]. Thoracic epidurals are recommended as standard practice for rib fractures [41], which is a common primary medical diagnosis or associated diagnosis in critically ill patients.

There is overwhelming evidence that thoracic epidurals provide improved pain and respiratory outcomes in critically ill, post-surgical patients. An alternative regional anesthetic technique is paravertebral blocks, which demonstrated similar pain reductions but with a lower incidence of complications compared to epidural techniques [42]. Recent evidence 
suggests that the combination of paravertebral blocks and subcostal transversus abdominis plane block may improve outcomes after minimally invasive esophagectomy. In 17 patients undergoing this surgery, the group receiving this combined regional anesthetic technique had less opioid consumption, less immediate postoperative pain, and shorter stay in the ICU [43].

\section{Complementary Treatments for Pain in Critically III Patients}

Alternative treatment modalities are also important supplements to pharmacological treatment of pain, especially with the multimodal approach to managing pain. They are cheap, safe, and easily available and can be routinely used in pain management in the ICU. However, evidence for their efficacy is generally limited.

Lack of sleep may impair cellular immunity and adversely affect tissue repair, whilst decreasing pain thresholds and increase stress levels $[44,45]$. Therefore, it is important to minimize stimuli that could disturb normal diurnal sleep patterns including noise, unpleasant ambient temperature, artificial lighting, frequent monitoring, or invasive procedures. Equally as important is the prompt treatment of anxiety and delirium [45]. Other commonly used techniques include a calming voice, relaxation techniques, deep breathing, music, distraction, and family presence. Music, deep breathing, and ice therapy showed inconsistent results in decreasing pain scores in the ICU $[46 \bullet, 47]$.

The increased susceptibility to pain and anxiety in the short-term can contribute to more serious chronic psychiatric disorders. Notably, critically ill patients are at an increased risk for developing depression and post-traumatic stress disorder $[48,49]$. One study found that one in four patients experience PTSD 1 year after discharge from ICU [50] and given that anxiety has been reported to be significantly associated with the risk for PTSD [51], controlling anxiety could help prevent long-term psychiatric complications. Pain and agitation are significant sources of distress in critically ill patients [52, 53], and the memory of distress due to a feeling of incapacitation has been shown to predict long-term symptoms of PTSD [50] in intensive care. Therefore, effective minimization of pain and anxiety could reduce the likelihood of longterm psychological co-morbidities.

Some level of psychological support has been shown to have a beneficial effect on health-related outcomes. For example, a strong social support is associated with fewer symptoms of PTSD and may foster better general mental stability among ICU patients [54]. Stable mental states are critical for the generation of useful coping strategies and can alleviate mental health disturbances. For example, it has been found that a pessimistic mindset is a predictor of PTSD symptoms, depression, and anxiety in critically ill patients [50]. Therefore, to encourage a feeling of positivity and improve psychological well-being, social support from family and caregivers should be encouraged where possible [51]. Other interventions such as guided imagery can also be a source of emotional support for patients [55].

The overall ICU experience can also be a predictor of PTSD symptoms at 1 year, and as this includes patients' memory of pain [50], careful control of pain during hospitalization could also prevent the development of future chronic psychiatric issues. Music therapy has been shown to reduce anxiety, which in turn can diminish the perception of pain $\left[20^{\bullet}, 46^{\bullet}\right]$ and could be beneficial to long-term psychological outcomes.

Another adverse outcome in critically ill patients in perioperative period is the occurrence of delirium. Delirium affects up to $87 \%$ of patients after admission to intensive care [56]. Hospitalacquired delirium is associated with an increased length of stay in intensive care and also heightens the risk of death during admission [57•]. Improving sleep using [58] and keeping interventions to a minimum during patients' normal hours of sleep [59] could help lower levels of anxiety. Effective pain management, breathing techniques, and improving mobility can also help prevent the onset of delirium [59].

\section{Conclusion}

Pain is a unique and vital aspect of the entire patient experience through the perioperative and intensive care unit setting. Multiple factors such as the patient themselves, pre-existing conditions including psychiatric history, the surgery or procedure being performed, and the recovery experience can all impact the type of pain one endures during recovery in the post-surgical ICU. Therefore, it is extremely vital to recognize this and accurately diagnose, assess, and manage pain of the critically ill in a timely manner. It is the medical teams' duty to recognize the importance of controlling pain and optimizing all conditions when possible to prevent central sensitization and chronic post-surgical pain, especially in the intensive care unit.

The diagnosis of pain in this particular patient group, however, presents multiple challenges for the clinician. Pain in ICU is difficult to assess and treat due to a variety of factors, but is largely due to the inability of the critically ill patient to adequately communicate and express their level of pain. Consequently, clinicians should have a low index of suspicion in assuming that the patient is in pain. Opioids are largely the mainstay of therapy for analgesia in critically ill patients, alongside other agents including ketamine, paracetamol, and where appropriate, NSAIDs. There is also a role for regional anesthesia techniques such as peripheral nerve blocks and epidurals as analgesic adjuncts in critically ill patients. 
Additionally, control of anxiety, stress, and sleep deprivation may lessen pain perception and lower analgesic requirements.

The improved outcomes in critically ill patients in perioperative period seen with optimized pain management may also have beneficial economic impacts on healthcare utilization. A recent retrospective cohort analysis examined the cost of ICU care and found that mechanical ventilation is associated with significantly higher daily costs for patients throughout their entire intensive care unit stay. The study suggested that interventions that reduced ICU length of stay and duration of mechanical ventilation could lead to substantial reductions in total inpatient cost [17•]. The importance of pre-emptive analgesic interventions such as thoracic epidurals and paravertebral techniques cannot be overstated, and the comprehensive assessment and management of pain is of key significance in this vulnerable group of patients.

\section{Compliance with Ethical Standards}

Conflict of Interest Michal Czernicki, Sreekumar Kunnumpurath, William Park, Anamika Kunnumpurath, Gopal Kodumudi, Jing Tao, Vijay Kodumudi, Nalini Vadivelu, and Richard D. Urman declare no conflict of interest.

Human and Animal Rights and Informed Consent This article does not contain any studies with human or animal subjects performed by any of the authors.

\section{References}

Papers of particular interest, published recently, have been highlighted as:

- Of importance

1. Klein DG, Dumpe M, Katz E, Bena J. Pain assessment in the intensive care unit: development and psychometric testing of the nonverbal pain assessment tool. Heart Lung. 2010;39(6):521-8. https:// doi.org/10.1016/j.hrtlng.2010.05.053.

2. Barr J, Fraser GL, Puntillo K, et al. Clinical practice guidelines for the management of pain, agitation, and delirium in adult patients in the intensive care unit. Crit Care Med. 2013;41(1):263-306. A very good review of the standard protocols and guidelines in assessing and diagnosing pain along with other common issues in the ICU.

3. Puntillo KA, White C, Morris AB, Perdue ST, Stanik-Hutt J, Thompson CL, et al. Patients' perceptions and responses to procedural pain: results from Thunder Project II. Am J Crit Care. 2001;10:238-51.

4. Szokol JW, Vender JS. Anxiety, delirium, and pain in the intensive care unit. Crit Care Clin. 2001;17(4):821-42.

5. Lewis KS, Whipple JK, Michael KA, Quebbeman EJ. Effect of analgesic treatment on the physiological consequences of acute pain. Am J Hosp Pharm. 1994;51:1539-54.

6. Chanques G, Jaber S, Barbotte E, Violet S, Sebbane M, Perrigault P, et al. Impact of systematic evaluation of pain and agitation in an intensive care unit. Crit Care Med. 2006;34:1691-9.
7. Payen JF, Bosson JL, Chanques G, Mantz J, Labarere J. DOLOREA investigators: pain assessment is associated with decreased duration of mechanical ventilation in the intensive care unit: a post hoc analysis of the DOLOREA study. Anesthesiology. 2009;111:1308-16.

8. Sricharoenchai T, Parker A, Raparla S, Schneck K, Bienvenu O and Needham D. A meta-analysis of post-traumatic stress disorder (PTSD) symptoms in intensive care unit Survivors, Am J Respir Crit Care Med. 2014;189(A2534).

9. Adair JC, Gilmore RL. Meperidine neurotoxicity after organ transplantation. J Toxicol Clin Toxicol. 1994;32(3):325-8.

10. Woolf CJ, Chong MS. Pre-emptive analgesia - treating postoperative pain by preventing establishment of central sensitization. Anesth Analg. 1993;77(2):362-79.

11. Pottecher J, Falcoz PE, Massard G, Dupeyron JP. Does thoracic epidural analgesia improve outcome after transplantation? Interact Cardiovasc Thorac Surg. 2011;12(1):51-3.

12. van Gulik L, Ahlers SJ, Brkić Z, Belitser SV, van Boven WJ, van Dongen EP, et al. Improved analgesia after the realization of a pain management programme in ICU patients after cardiac surgery. Eur J Anaesthesiol. 2010;27(10):900-5.

13. Kelly DJ, Ahmad M, Brull SJ. Pre-emptive analgesia 1; physiological pathways and pharmacological modalities. Can J Anaesth. 2001;48(10). Provides a detailed explanation on defining preemptive analgesia and how it can affect acute post-surgical pain. It also leads into how this type of pain can develop into chronic post surgical pain and the pharmacological modalities involved to help prevent this complication.

14. Kelly DJ, Ahmad M, Brull SJ. Pre-emptive analgesia 1; physiological pathways and pharmacological modalities. Can J Anaesth. 2001;48(10).

15. Suzuki H. Recent topics in the management of pain: development of the concept of pre-emptive analgesia. Cell Transplant. 1995;4(Suppl 1):S3.

16. Morphine pellet-induced immunomodulation in mice: temporal relationships. J Pharmacol Exp Ther. 1988;245(3):913-20.

17. Rose K, Meibner W. Pain therapy in intensive care patients. Anaethesist. 2018;67(6):401-8. https://doi.org/10.1007/s00101018-0458-x. Most recent summary on pain management techniques in the ICU specifically.

18. Macintyre PE, Ready LB. Acute pain management a practical guide. 2nd ed. London: WB Saunders; 2002.

19. Eddleston JM, White P, Guthrie E. Survival, morbidity, and quality of life after discharge from intensive care. Crit Care Med. 2000;28: 2293-9. https://doi.org/10.1097/00003246-200007000-00018. Offers insight into what it means for patients after an ICU stay. Quality of life is essential in all parts of a patient's medical journey and the recovery after an ICU stay can be challenging and even lead to morbidity, mortality, or death.

20. Chlan L, Halm MA. Does music ease pain and anxiety in the critically ill? Am J Crit Care. 2013;22(6):528-32. Explores other holistic modalities to help treat pain and its psych ological impact. This continues to support the multimodal approach and also suggests that not only medical, pharmacological, and interventional approaches can help patients' symptoms.

21. Ekman E, Koman A. Acute pain following musculoskeletal injuries and orthopaedic surgery. J Bone Joint Surg. 2004;86-A:1316-24.

22. Macintyre PE, Ready LB. Acute pain management a practical guide. 2nd ed. London: WB Saunders; 2002.

23. Eddleston JM, White P, Guthrie E. Survival, morbidity, and quality of life after discharge from intensive care. Crit Care Med. 2000;28: 2293-9. https://doi.org/10.1097/00003246-200007000-00018.

24. Chanques G, Jaber S, Barbotte E, Violet S, Sebbane M, Perrigault $\mathrm{PF}$, et al. Impact of systematic evaluation of pain and agitation in an intensive care unit. Crit Care Med. 2006;34(6):1691-9. 
25. Li D, Puntillo K, Miaskowski C. A review of objective pain measures for use with critical care adult patients unable to self-report. J Pain. 2008;9(1):2-10.

26. Chanques G, Viel E, Constantin JM, Jung B, de Lattre S, Carr J, et al. The measurement of pain in intensive care unit: comparison of 5 self-report intensity scales. Pain. 2010;151(3):711-21.

27. Li D, Puntillo K, Miaskowski C. A review of objective pain measures for use with critical care adult patients unable to self-report. J Pain. 2008;9(1):2-10.

28. Shannon K, Bucknall T. Pain assessment in critical care: whathavewe learnt from research. Intensive Crit Care Nurs. 2003;19(3):154-62.

29. Caswell DR, Williams JP, Vallejo M, Zaroda T, McNair N, Keckeisen M, et al. Improving pain management in critical care. Jt Comm J Qual Improv. 1996;22(10):702-12.

30. Erstad BL, Puntillo K, Gilbert HC, Grap MJ, Li D, Medina J, et al. Pain management principles in the critically ill. Chest. 2009;135(4): 1075-86.

31. Murray MJ, Plevak DJ. Analgesia in the critically ill patient. New Horiz. 1994;2(1):56-63.

32. Bryant HU, Bernton EW, Holaday JW. Distinction between the in vitro and in vivo inhibitory effects of morphine on lymphocyte proliferation based on agonist sensitivity and naltrexone reversibility. Immunopharmacology. 1992;23(2):117-24 Bayer BMGastonguay MRHernandez MC:Abstract.

33. Cammarano WB, Pittet JF, Weitz S, Schlobohm RM, Marks JD. Acute withdrawal syndrome related to the administration of analgesia and sedative medications in adult intensive care unit patients. Crit Care Med. 1998;26(4):676-84.

34. Pandey CK, Bose N, Garg G, Singh N, Baronia A, Agarwal A, et al. Gabapentin for the treatment of pain in guillain-barré syndrome: a double-blinded, placebo-controlled, crossover study. Anesth Analg. 2002;95(6):1719-23.

35. Zhang J, Ho KY, Wang Y. Efficacy of pregabalin in acute postoperative pain: a meta-analysis. Br J Anaesth. 2011;106(4):454-62. https://doi.org/10.1093/bja/aer027.

36. Koppert W, Weigand M, Neumann F, Sittl R, Schuettler J, Schmelz $\mathrm{M}$, et al. Perioperative intravenous lidocaine has preventive effects on postoperative pain and morphine consumption after major abdominal surgery. Anesth Analg. 2004;98(4):1050-5.

37. Harvey KP, Adair JD, Isho M, Robinson R. Can intravenous lidocaine decrease postsurgical ileus and shorten hospital stay in elective bowel surgery? A pilot study and literature review. Am J Surg. 2009;198(2):231-6

38. Loftus RW, Yeager MP, Clark JA, Brown JR, Abdu WA, Sengupta DK, et al. Intraoperative ketamine reduces perioperative opiate consumption in opiate-dependent patients with chronic back pain undergoing back surgery. Anesthesiology. 2010;113(3):639-46.

39. Laskowski K, Stirling A, McKay WP, Lim HJ. A systematic review of intravenous ketamine for postoperative analgesia. Can J Anaesth. 2011;58(10):911-23.

40. Hebl JR, Dilger JA, Byer DE, Kopp SL, Stevens SR, Pagnano MW, et al. A pre-emptive multimodal pathway featuring peripheral nerve block improves perioperative outcomes after major orthopedic surgery. Reg Anesth Pain Med. 2008;33(6):510-7.

41. Ilfeld BM. Continuous peripheral nerve blocks: a review of the published evidence. Anesth Analg. 2011;113(4):904-25.

42. Nishimori M, Low JH, Zheng H, Ballantyne JC. Epidural pain relief versus systemic opioid-based pain relief for abdominal aortic surgery. Cochrane Database Syst Rev. 2012;7:CD005059.

43. Manion SC, Brennan TJ. Thoracic epidural analgesia and acute pain management. Anesthesiology. 2011;115(1):181-8.
44. Simon BJ, Cushman J, Barraco R, et al. EAST Practice Management uidelines ork roup. Pain management guidelines for blunt thoracic trauma. J Trauma. 2005;59(5):1256-67.

45. Davies RG, Myles PS, Graham JM. A comparison of the analgesic efficacy and side-effects of paravertebral vs epidural blockade for thoracotomy - a systematic review and meta-analysis of randomized trials. Br J Anaesth. 2006;96(4):418-26.

46. Li NL, Liu CC, Cheng SH, et al. Feasibility of combined paravertebral block and subcostal transversus abdominis plane block in postoperative pain control after minimally invasive esophagectomy. Acta Anaesthesiol Taiwan. 2013;51(3):103-7. Good review on regional techniques as adjuncts to multimodal analgesia in post-surgical pain.

47. Griffiths J, Fortune G, Barber V, Young JD. The prevalence of posttraumatic stress disorder in survivors of ICU treatment: a systematic review. Intensive Care Med. 2007;33:1506-18.

48. Scragg P, Jones A, Fauvel N. Psychological problems following ICU treatment. Anaesthesia. 2001;56:9-14. https://doi.org/10. 1046/j.1365-2044.2001.01714.x.

49. Myhren H, Ekeberg O, Toien K, Karlsson S, Stokland O. Posttraumatic stress, anxiety and depression symptoms in patients during the first year post intensive care unit discharge. Crit Care. 2010;14(1):R14.

50. Deja M, Denke C, Weber-Carstens S, Schröder J, Pille CE, Hokema $\mathrm{F}$, et al. Social support during intensive care unit stay might improve mental impairment and consequently health-related quality of life in survivors of severe acute respiratory distress syndrome. Crit Care. 2006; 10:R147.

51. Breckenridge SJ, Chlan L, Savik K. Impact of tracheostomy placement on anxiety in mechanically ventilated adult ICU patients. Heart \& Lung. 2014;43(5):392-8.

52. Chanques G, Jaber S, Barbotte E, Violet S, Sebbane M, Perrigault $\mathrm{PF}$, et al. Impact of systematic evaluation of pain and agitation in an intensive care unit. Crit Care Med. 2006;34(6):1691-9.

53. Schnyder U, Morgeli H, Nigg C, Klaghofer R, Renner N, Trentz O, et al. Early psychological reactions to life-threatening injuries. Crit Care Med. 2000;28:86-92. https://doi.org/10.1097/00003246200001000-00014.

54. Halpin LS, Speir AM, CapoBianco P, Barnett SD. Guided imagery in cardiac surgery. Outcomes Manag. 2002;6:132-7.

55. Rea RS, Battistone S, Fong JJ, Devlin JW. Atypical antipsychotics versus haloperidol for treatment of delirium in acutely ill patients. Pharmacotherapy. 2007;27(4):588-94.

56. Shehabi Y, Riker RR, Bokesch PM, Wisemandle W, Shintani A, Ely EW. Delirium duration and mortality in lightly sedated, mechanically ventilated intensive care patients. Crit Care Med. 2010;38(12):2311-8.

57. Van Rompaey B, Elseviers MM, Van Drom W, Fromont V, Jorens PG. The effect of earplugs during the night on the onset of delirium and sleep perception: a randomized controlled trial in intensive care patients. Crit Care. 2012;16(3):R73. Investigates another way to help treat the dreadful ICU delirium besides using pharmacological methods which has its own side effects. The article suggests that using all tools available to help the sleep-wake cycle could help prevent and treat ICU delirium.

58. Brummel NE, Girard TD. Preventing delirium in the intensive care unit. Crit Care Clin. 2013;29(1):51-65.

59. Dasta JF, McLaughlin TP, Mody SH, et al. Daily cost of an intensive care unit day: the contribution of mechanical ventilation. Crit Care Med. 2005;33(6):1266-71.

Publisher's Note Springer Nature remains neutral with regard to jurisdictional claims in published maps and institutional affiliations. 